\title{
Descontaminación de agua utilizando nanomateriales y procesos fotocatalíticos
}

\author{
Juan Carlos Durán-Álvarez, ${ }^{*}$ Edwin Avella, ${ }^{*}$ Rodolfo Zanella*
}

RESUMEN: La efectiva eliminación de agentes contaminantes en el agua es un tema de prioridad en todo el mundo. Muchas de estas sustancias contaminantes son recalcitrantes en los sistemas convencionales de tratamiento de agua, por lo que un importante número de sistemas de tratamiento avanzado ha sido desarrollado. Los procesos de oxidación avanzada, como la fotocatálisis heterogénea, han demostrado eliminar eficientemente un amplio grupo de agentes contaminantes de carácter orgánico, inorgánico y microbiológico presentes en el agua contaminada. En este artículo, se revisan los principios básicos del proceso de fotocatálisis heterogénea, así como los mecanismos involucrados en la degradación de contaminantes de diferente tipo en el agua. Adicionalmente, se hace una revisión del impacto que puede tener la modificación en superficie de semiconductores sobre la eficiencia de los procesos fotocatalíticos.

PALABRAS CLAVE: contaminantes, fotocatálisis, nanopartículas metálicas, óxidos metálicos, semiconductores.

ABSTRACT: The removal of water pollutants is a priority issue worldwide. Many of these contaminants are recalcitrant in conventional water and wastewater treatment systems; due to this, a significant number of advanced treatment systems have been developed in order to efficiently remove such substances from water. Advanced oxidation processes such as heterogeneous catalysis have shown to efficiently remove a large group of organic and inorganic pollutants as well as microbiological agents. In this article, the principles of heterogeneous photocatalysis process are reviewed as well as the mechanisms involved in the degradation of contaminants in the water. Additionally, a brief review of the impact that surface modification of semiconductors have on the performance of the photocatalytic process is given.

KEYWORDS: metallic nanoparticles, metallic oxides, photocatalysis, pollutants, semiconductors.

\section{Introducción}

La presencia de contaminantes en el agua es un tema de prioridad internacional, pues no sólo se relaciona con la salud de los ecosistemas, sino con el abastecimiento de agua para los individuos y el desarrollo de diversas actividades económicas. Por lo anterior, preservar la buena calidad del agua se ha

* Centro de Ciencias Aplicadas y Desarrollo Tecnológico (CCADET), Universidad Nacional Autónoma de México (UNAM), México D. F., México A. P. 70-186. Correspondencia: Rodolfo Zanella, Tel.: +52 55 5622-8601, Fax. +52 55 5550-0654 (rodolfo.zanella@ccadet. unam.mx).

Los autores agradecen el financiamiento de CONACYT-México a través de los proyectos 130407 y 163098 , así como de la DGAPA-UNAM por el financiamiento otorgado a través del proyecto 103513 . 
convertido en un tema tanto de seguridad nacional, en algunos países, como en una de las metas del milenio establecidas por la Organización de las Naciones Unidas. Los agentes contaminantes del agua pueden ser de carácter orgánico, inorgánico o microbiológico, y éstos pueden tener un origen natural o antropogénico. Por ejemplo, la disolución de los minerales contenidos en el material del acuífero puede contaminar los cuerpos de agua subterránea con metales pesados, tales como el arsénico o el cromo, mientras que las descargas de agua residual de las ciudades son la principal fuente de contaminación de ríos y lagos. Debido a la continua entrada de los contaminantes a los cuerpos de agua y a que algunos de ellos pueden ser persistentes, se ha desarrollado una amplia variedad de sistemas de tratamiento, los cuales pueden ser aplicados de manera individual o en conjunto para alcanzar la calidad deseada en el agua tratada. La selección del sistema de tratamiento depende, en gran medida, del propósito definido para el agua tratada; de ahí que los procesos biológicos sean comúnmente seleccionados para remover la mayor cantidad de material orgánico de las aguas residuales, mientras otros procesos como la coagulación-floculación-filtración-desinfección sean los de uso típico para potabilizar el agua subterránea. Aun cuando existe una larga lista de sistemas de tratamiento de agua, cada uno basado en procesos como la filtración, la biodegradación, la oxidación y la adsorción, existe un importante número de agentes contaminantes recalcitrantes a los diferentes procesos de tratamiento, ya que son poco biodegradables, afines a los lodos residuales o resistentes a los procesos de desinfección. Para estos casos se han desarrollado procesos avanzados de tratamiento, lo suficientemente agresivos para transformar, degradar o inactivar los agentes contaminantes de mayor recalcitrancia.

Los procesos de oxidación avanzada (POA) son más efectivos para la eliminación de contaminantes recalcitrantes que los sistemas convencionales de tratamiento de agua (Malato et al., 2009). Estos métodos están basados en la formación de especies químicas altamente reactivas y de baja selectividad, tales como los radicales hidroxilo $\left({ }^{\bullet} \mathrm{OH}\right)$ y superóxido $\left({ }^{\circ} \mathrm{O}_{2}{ }^{-}\right)$o el peróxido de hidrógeno $\left(\mathrm{H}_{2} \mathrm{O}_{2}\right)$. Los POA han mostrado ser eficientes para la remoción de sustancias recalcitrantes presentes en el agua a muy bajas concentraciones (Comniellis et al., 2008; Oller et al., 2011). En la mayoría de los casos, estos procesos consumen cantidades mínimas de energía y son capaces de mineralizar ciertos contaminantes orgánicos refractarios (Lastre et al., 2012). Entre los procesos de oxidación avanzada, la fotocatálisis heterogénea ha mostrado ser muy efectiva en el tratamiento del agua. Este proceso consiste en reducir la energía de activación de una reacción fotoquímica mediante el uso de un fotocatalizador en estado sólido; es decir, el proceso se lleva a cabo en la interfase sólido-líquido. El agente fotocatalizador es capaz de producir transformaciones químicas de los reactivos mediante interacciones repetidas entre ellos, para regenerarse después de cada ciclo de interacciones. Las ventajas de este proceso estriban en: a) eliminar los agentes 
contaminantes del agua en periodos cortos con mínima generación de residuos (por ejemplo, lodos) y baja generación de subproductos de degradación; b) alcanzar altas tasas de desinfección en poco tiempo, junto con bajos índices de recrecimiento de los agentes microbianos; c) requieren bajas cantidades de reactivo para llevar a cabo el proceso de fotocatálisis, y en la mayoría de los casos es posible recuperar y reusar al catalizador; d) es posible modificar al fotocatalizador para ser activado con luz solar, y, e) es posible conjugar varios procesos de oxidación avanzada para obtener sistemas híbridos que trabajen más eficientemente por medio de la sinergia de sus componentes individuales. En las últimas dos décadas, se ha trabajado de manera intensiva en la modificación de los materiales fotocatalíticos, con el fin de incrementar su actividad en el espectro de luz visible. Esto traería como resultado el potencial uso de los fotocatalizadores en plantas solares de tratamiento de agua, lo cual sería favorecedor para la implementación de esta clase de sistemas en zonas con alta irradiación solar, como es el caso de la mayor parte de los países latinoamericanos.

En este artículo se exploran los aspectos más relevantes acerca de los principios de la fotocatálisis heterogénea, así como el desarrollo e implementación de materiales nanoestructurados para la eliminación fotocatalítica de agentes contaminantes en el agua. Se describirán algunas de las modificaciones estructurales realizadas a los nanomateriales fotocatalíticos para: a) incrementar su actividad como fotocatalizador; b) favorecer su actividad fotocatalítica en el espectro de luz visible o luz solar, y, c) reducir su desactivación.

\section{Uso de materiales nanoestructurados en fotocatálisis heterogénea}

A principios del siglo XX, Ostwald definió un catalizador como "una sustancia que incrementa la velocidad a la cual un sistema químico se aproxima al equilibrio, sin ser consumida en el proceso" (Polshettiwar y Asefa, 2013). Un fotocatalizador es aquella sustancia que se activa químicamente al ser irradiada con luz a una cierta longitud de onda o en un rango de longitudes de onda. Tradicionalmente, los fotocatalizadores se dividen en dos grandes grupos: los fotocatalizadores homogéneos y los heterogéneos. Los primeros son aquellos que existen en la misma fase que los reactivos, por ejemplo, sales metálicas y complejos orgánicos u organometálicos; estos fotocatalizadores son generalmente difíciles de separar de la mezcla de reacción para su reuso. Por otra parte, los fotocatalizadores heterogéneos son sólidos insolubles, como la ceria, la alúmina o la titania, y, debido a ello, son fácilmente separables y reusables al final del proceso fotocatalítico en agua. Los materiales nanométricos, tales como las nanopartículas metálicas y los semiconductores, son capaces de actuar como fotocatalizadores heterogéneos de alta actividad y estabilidad, pero también como soportes de fases aún más activas, o bien 
FIGURA 1. Representación esquemática del proceso fotocatalítico empleando un semiconductor.

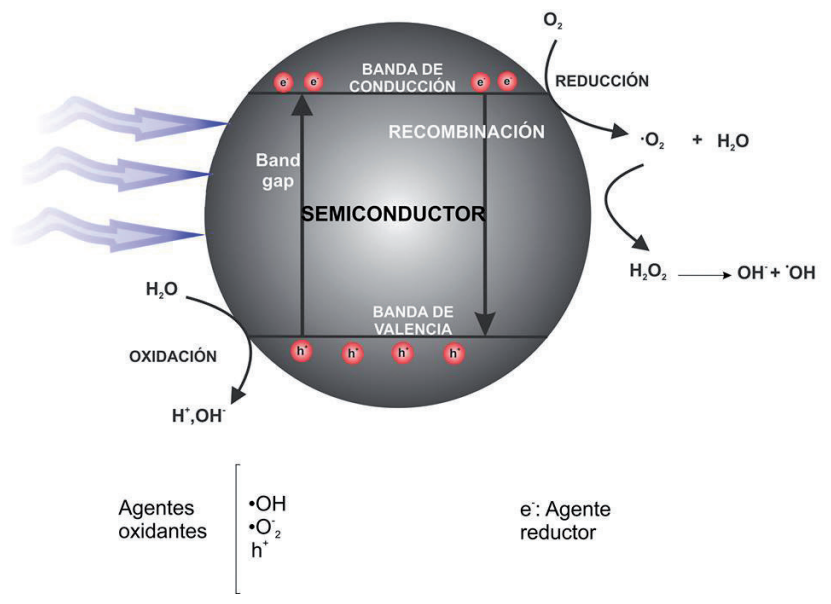

Fuente: Elaboración propia.

como cocatalizadores (Astruc, 2008). Cuando los fotocatalizadores son de tamaño nanométrico pueden ser considerados como materiales intermedios entre lo homogéneo y lo heterogéneo debido a sus dimensiones diminutas. Estos materiales presentan altas áreas específicas y un elevado número de sitios activos expuestos - por tanto, existe una mayor área de contacto con los reactantes-, esto los hace parecidos en cierta medida a los catalizadores homogéneos. Al mismo tiempo, los fotocatalizadores nanoestructurados son efectivamente catalizadores heterogéneos al estar en una fase diferente al medio de reacción (en este caso, el agua) y por ello tienen el potencial de ser separados y reusados en varios ciclos. La dualidad presentada por estos nanomateriales permite diseñar fotocatalizadores con mayor actividad, estabilidad y selectividad mediante la manipulación de su síntesis, logrando la modificación de su tamaño, estructura y morfología (Hashmi y Hutchings, 2006; Polshettiwar y Asefa, 2013).

De manera general, se puede decir que el proceso de fotocatálisis en fase heterogénea está basado en reacciones de oxidación-reducción, las cuales ocurren en la superficie del fotocatalizador. Cuando se emplean semiconductores como fotocatalizador, el paso inicial del proceso consiste en la absorción de energía lumínica por parte del material nanoestructurado; esta energía deberá ser suficiente para excitar a los electrones en la banda de valencia, promoviéndolos hacia la banda de conducción (figura 1). Ello genera el par hueco-electrón, término que se refiere a la deficiencia de electrones en la banda de menor energía (banda de valencia) y la presencia simultánea de un electrón excitado en la banda de conducción. Al mantenerse la separación de cargas, el hueco $\left(\mathrm{h}^{+}\right)$y el electrón $\left(\mathrm{e}^{-}\right)$pueden migrar a la superficie del catalizador, en donde inician una cascada de reacciones de oxidación y reduc- 
ción, en las cuales participan las especies químicas adsorbidas en la superficie del catalizador (Muneer et al., 2005).

Los huecos foto-formados en la superficie del semiconductor presentan carga positiva y pueden oxidar a las moléculas de agua o a cualquier otro componente adsorbido en el catalizador, y con ello generar especies altamente oxidantes, tal y como se muestra en las ecuaciones 1 y 2 :

$$
\begin{aligned}
\mathrm{h}^{+}+\mathrm{H}_{2} \mathrm{O}_{(\mathrm{ads})} & \longleftrightarrow \mathrm{H}_{2} \mathrm{O}^{+}{ }_{(\mathrm{ads})} \\
\mathrm{h}^{+}+\mathrm{OH}^{-}{ }_{(\mathrm{ads})} & \longleftrightarrow \cdot \mathrm{OH}_{(\mathrm{ads})}+\mathrm{H}^{+}
\end{aligned}
$$

Una vez formados, los radicales hidroxilo activados $(\cdot \mathrm{OH})$ actúan como agentes oxidantes fuertes. Por otro lado, los electrones promovidos hacia la banda de conducción del semiconductor reaccionan con las moléculas de oxígeno adsorbidas para producir el radical superóxido $\left({ }^{\circ} \mathrm{O}_{2}{ }^{-}\right)$, como se muestra en la ecuación 3. Estos radicales son rápidamente convertidos en peróxido de hidrógeno (ecuación 4) y, posteriormente, en radicales hidroxilo activados (ecuación 5).

$$
\begin{aligned}
\mathrm{e}^{-}+\mathrm{O}_{2(\mathrm{ads})} & \longleftrightarrow \cdot \mathrm{O}_{2}^{-}{ }_{(\mathrm{ads})} \\
\cdot \mathrm{O}_{2}^{-}{ }_{(\mathrm{ads})}+2 \mathrm{H}^{+} & \longleftrightarrow \mathrm{H}_{2} \mathrm{O}_{2(\mathrm{ads})} \\
\mathrm{e}^{-}+\mathrm{H}_{2} \mathrm{O}_{2(\mathrm{ads})} & \longleftrightarrow \cdot \mathrm{OH}_{(\mathrm{ads})}+\mathrm{OH}^{-}{ }_{(\mathrm{ads})}
\end{aligned}
$$

La eficiencia de las reacciones arriba descritas depende de cuatro procesos, a saber: a) la absorción de luz por el semiconductor; b) la separación del par hueco-electrón; c) la migración de los pares foto-producidos hacia la superficie del catalizador, y, d) la velocidad de recombinación de los pares hueco-electrón que se hayan foto-formado (Cushing et al., 2012).

La eficiencia del transporte del electrón entre las bandas de valencia y de conducción se ve fuertemente afectada por la estructura cristalina y el tamaño de partícula del fotocatalizador. Por ejemplo, un material cristalino que contiene únicamente pequeñas cantidades de defectos estructurales - esto es, deficiencias en la red de átomos que conforman el cristal - puede suprimir la recombinación de los pares hueco-electrón y, consecuentemente, aumentar el tiempo de vida de las especies foto-generadas (Osterloh, 2008). Una de las ventajas de usar fotocatalizadores nanométricos es que los electrones deben migrar una distancia muy pequeña hacia la superficie del semiconductor, factor que, se estima, también contribuye a disminuir la probabilidad de recombinación. De hecho, se sabe que la energía de banda prohibida - esto es, la diferencia de energía entre la banda de valencia y la de conducción- y otras propiedades fisicoquímicas del semiconductor pueden cambiar si se disminuye el tamaño de partícula o se modifica la forma de las 
FIGURA 2. Representación esquemática de la resonancia superficial del plasmón en nanopartículas de metales nobles (esquema superior), direccionalidad de la transferencia de electrones en nanopartículas metálicas con diferente morfología (esquema inferior).

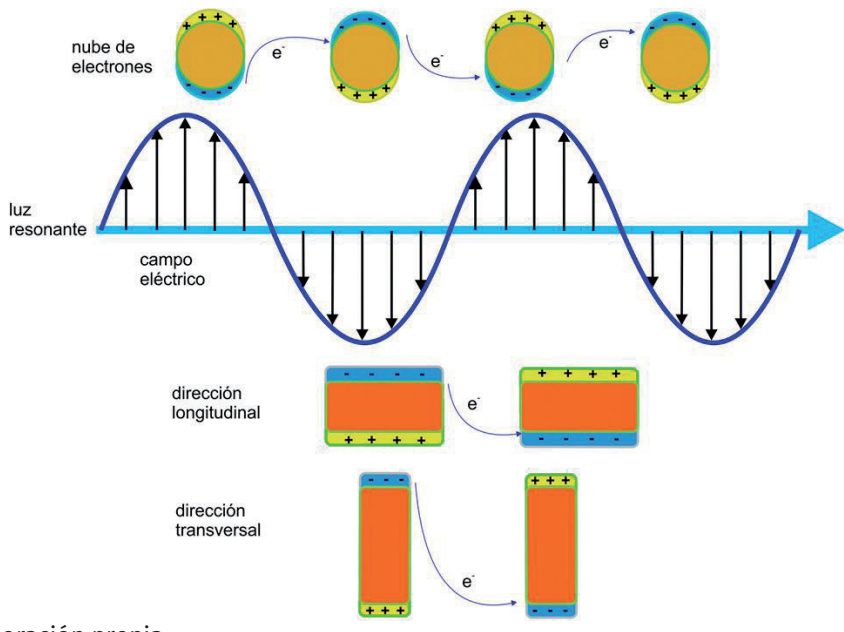

Fuente: Elaboración propia.

partículas nanométricas (Lin et al., 2009). Debido a que los diferentes semiconductores requieren un nivel específico de energía para alcanzar la transferencia entre bandas del electrón, éstos pueden ser catalogados en dos grupos: los que pueden ser activados sólo con luz UV (por ejemplo, $\mathrm{TiO}_{2}$, $\mathrm{ZnO}, \mathrm{SiO}_{2}$ y $\mathrm{Al}_{2} \mathrm{O}_{3}$ ), y los que son activos en el espectro de luz UV-visible (por ejemplo, $\mathrm{WO}_{3}, \mathrm{CeO}_{2}, \mathrm{Fe}_{2} \mathrm{O}_{3}, \mathrm{Bi}_{2} \mathrm{O}_{3}, \mathrm{BiVO}_{4}$ y $\mathrm{CdS}$ ) debido a su bajo valor de energía de banda prohibida. A este respecto, es importante destacar que aun cuando algunos fotocatalizadores pueden activarse con luz visible, ello no necesariamente se traduce en una alta actividad fotocatalítica en esta zona del espectro, pues algunos de estos materiales presentan un alto grado de recombinación del par hueco-electrón, inactivándose antes de generar radicales libres y especies oxidantes en la superficie del semiconductor.

Por otra parte, cuando en la fotocatálisis heterogénea se emplean nanopartículas de metales nobles — por ejemplo, Au, Ag, Pt, Pd, Ir-, la cascada de reacciones de oxidación-reducción se inicia con la absorción de luz por las nanopartículas metálicas a una longitud de onda determinada. Los electrones en la banda de conducción de las nanopartículas del metal conductor son excitados por acción de la resonancia superficial del plasmón; fenómeno descrito como los movimientos oscilatorios de los electrones en la banda de conducción de un metal noble por acción de la incidencia de luz visible (figura 2). Posteriormente, las nanopartículas metálicas generan un campo eléctrico muy intenso cerca de su superficie. Se ha demostrado que la intensidad del campo eléctrico localizado en los "puntos calientes" plasmónicos puede ser hasta 1,000 veces superior a la del campo eléctrico incidente (Hou 
Tabla 1. Longitudes de onda de banda del plasmón reportadas para algunos metales.

\begin{tabular}{|l|c|l|}
\hline METAL & LONGITUD DE ONDA (nm) & REFERENCIA \\
\hline Oro & 552 & Eustis y El-Sayed (2006) \\
\hline Plata & 480 & You et al. (2009) \\
\hline Cobre & 578 & Gwak et al. (1998) \\
\hline Platino & 420 & You et al. (2009) \\
\hline Níquel & 400 & Chen et al. (2011) \\
\hline
\end{tabular}

Fuente: Elaboración propia.

y Cronin, 2013; Le et al., 2008; Zou y Schatz, 2005). Debido a ello, la velocidad de generación de los pares hueco-electrón en estos "puntos calientes" es 1,000 veces superior a lo observado en otras regiones de la nanopartícula (Hou y Cronin, 2013). Dado que el campo está confinado a unos cuantos nanómetros en la superficie de las nanopartículas, la mayoría de los pares hueco-electrón inducidos por el plasmón participan en el proceso fotocatalítico a través de las reacciones de oxidación-reducción arriba descritas (Hou y Cronin, 2013). La longitud de onda a la cual se expresa la resonancia plasmónica depende fuertemente del tipo y tamaño de las nanopartículas, así como de las propiedades dieléctricas del medio. Un caso excepcional son las nanopartículas de oro, las cuales presentan absorción tanto en el espectro visible como en el ultravioleta; esto último debido a las transiciones electrónicas de las bandas $5 d$ hacia las bandas $6 s p$ (Zhu et al., 2009). En la tabla 1, se muestran las longitudes de onda de la banda para las cuales algunos metales presentan resonancia superficial del plasmón.

La aplicación de la resonancia superficial del plasmón en los procesos de degradación de contaminantes en agua ha sido ampliamente estudiada durante las últimas tres décadas (Pradeep, 2009), siendo el oro el metal noble que presenta la resonancia plasmónica más intensa en comparación con otros metales nobles como plata, cobre o níquel (Primo et al., 2011). Es importante tomar en consideración que la intensidad de la resonancia superficial del plasmón puede variar en función de propiedades físicas de la nanopartícula metálica, tales como su tamaño o morfología. El efecto de resonancia del plasmón puede ser longitudinal o transversal, dependiendo de la longitud y orientación de la nanopartícula metálica (ver figura 2). Por esta razón, continuamente se están desarrollando técnicas de síntesis que permitan manipular las propiedades morfológicas de las nanopartículas metálicas y con ello lograr diferentes efectos en la resonancia superficial del plasmón.

El uso de materiales fotocatalíticos conjugados en el proceso de fotocatálisis heterogénea ha mostrado tener resultados prometedores en la depuración del agua. Un ejemplo de materiales nanométricos conjugados son los semiconductores modificados en superficie por el depósito de nanopartículas de metales nobles. Esta modificación puede resultar en un incremento de la 
fotoactividad del material híbrido con relación a sus componentes individuales; ello ocurrirá siempre y cuando las nanopartículas metálicas presenten propiedades plasmónicas (Cao et al., 2011). Otro ejemplo de estos sistemas son los semiconductores modificados en superficie por la adsorción de moléculas orgánicas con propiedades fotoquímicas, las cuales pueden alcanzar el estado de triplete a una longitud de onda similar a la del semiconductor y favorecer la activación del fotocatalizador mediante la transferencia de electrones desde la molécula excitada hacia la banda de conducción del semiconductor (Chong et al., 2010). En tercer lugar, se pueden mencionar los sistemas binarios o ternarios, los cuales se componen de dos semiconductores con diferente energía de banda prohibida, o bien, dos semiconductores modificados en superficie con nanopartículas metálicas. Estos compositos requieren menor energía de activación que sus componentes individuales, por lo cual son más eficientes.

Para que los materiales fotocatalíticos sean duraderos, éstos deben ser químicamente inertes, poco corrosibles y resistentes a la fotoxidación. Por esta razón, los óxidos metálicos y los metales nobles son los más adecuados para ser usados como fotocatalizadores en procesos de depuración de agua (Li et al., 2008). El dióxido de titanio, por ejemplo, tiene un muy bajo potencial de corrosión durante los procesos fotocatalíticos en matrices acuosas, además de tener bajo potencial de toxicidad y ser de bajo costo. Es por ello que es hasta ahora uno de los materiales más empleados en los sistemas de tratamiento de agua por fotocatálisis heterogénea.

\section{Fotocatálisis heterogénea usando nanomateriales para la depuración del agua}

Como se mencionó anteriormente, la fotocatálisis heterogénea se basa en una cascada de reacciones de oxidación-reducción, iniciada por la excitación y transferencia interbanda de electrones en un material sólido. Uno de los campos de mayor desarrollo para este proceso en los últimos años es el aprovechamiento y potenciación de dichas reacciones para la degradación de contaminantes en el agua. Esto entraña una serie de prerrogativas tales como: a) el incremento en la eficiencia de degradación o inactivación de agentes contaminantes en comparación con el proceso de fotólisis (Malato et al., 2009); b) la completa degradación de los contaminantes orgánicos hasta sus componentes minerales (Oller et al., 2011), y, c) el potencial de combinar a las reacciones de oxidación-reducción en procesos mixtos que involucren la degradación de contaminantes de diferente naturaleza (por ejemplo, oxidación de compuestos orgánicos acoplado a la reducción de metales pesados) junto con la generación de productos de muy alto valor energético (producción de hidrógeno por el rompimiento de la molécula de agua) (Patsoura et al., 2007; Jungwon y Choi, 2010).

La mayor parte de los estudios que reportan la eliminación de contami- 
nantes en el agua se han llevado a cabo bajo condiciones ideales, empleando agua destilada en un sistema de reacción discontinuo o semicontinuo y con una fuente de luz artificial. Esto se debe a que al trabajar con agua no ideal, tal como agua de lago, agua potable o agua residual tratada, se introducen en el proceso una serie de variables que dificultan el estudio cinético y el escalamiento del proceso desde las condiciones ideales hasta llegar a un sistema lo más cercano posible a una planta piloto. Los estudios más recientes reportan el tratamiento fotocatalítico de aguas naturales o de agua residual sintética enriquecidas con el contaminante objetivo (Soares et al., 2014; Wang et al., 2015). Más aún, Wang et al. (2015) reportan el desempeño de un fotocatalizador de $\mathrm{ZnO}$ modificado en superficie con nanopartículas de plata para la degradación de hormonas sexuales en agua residual no sintética; estos autores tratan de explicar el efecto que algunos de los componentes de la matriz tienen sobre la reacción de fotocatálisis. Así pues, es importante destacar que una serie de estudios previos bajo condiciones ideales tuvieron que realizarse para llegar a trabajar con matrices acuosas no ideales.

A continuación se describen a grandes rasgos los procesos que ocurren en la eliminación de diferentes clases de contaminantes durante la fotocatálisis heterogénea.

\section{Degradación de contaminantes orgánicos del agua}

La degradación de contaminantes orgánicos en el agua es uno de los temas más estudiados en el campo de la fotocatálisis heterogénea. Ello se debe, por un lado, a la enorme variedad de sustancias orgánicas que llegan a los cuerpos de agua y que son de relevancia ambiental debido a los efectos nocivos que causan a los organismos expuestos. Asimismo, la degradación de compuestos orgánicos en agua es uno de los métodos más empleados para probar el desempeño de los nuevos fotocatalizadores.

La degradación de compuestos orgánicos coloridos, como los colorantes azo, ha sido ampliamente reportada para diferentes fotocatalizadores (Akpan y Hameed, 2009; Rauf y Ashraf, 2009); éste es un sistema de prueba sencillo y fiable, la no requerir de métodos de análisis sofisticados. En el caso de los contaminantes orgánicos no coloridos, los estudios más recientes se han enfocado en la degradación fotocatalítica de los llamados contaminantes emergentes, definidos como sustancias químicas contenidas en productos de uso común, tales como los medicamentos, los productos de aseo personal, los aditivos que dan flexibilidad a los plásticos o aquellos que son precursores del teflón, las fragancias, los anticonceptivos, los tensoactivos, entre muchos otros (Daughton, 2004). Debido a su modo de uso, estas sustancias químicas son desechadas principalmente a través del drenaje y por ello son ubicuos en el agua residual, en los ríos y lagos, y hasta en el agua subterránea (Pal et al., 2010; Deblonde et al., 2011; Lapworth et al., 2012). Aun cuando las concentraciones de contaminantes emergentes están en niveles de trazas, 
éstos pueden causar efectos sutiles y crónicos en las especies acuáticas expuestas (Farré et al., 2008), tales como la feminización de poblaciones de peces y anfibios (Nagahama et al., 2003), o el desarrollo de resistencia a antibióticos por parte de organismos patógenos (Rodríguez-Mozaz et al., 2015). Es por ello que la remoción de los contaminantes emergentes del agua es una prioridad a todos los niveles.

En términos generales, la degradación fotocatalítica de los contaminantes orgánicos es un proceso de oxidación, producido por los radicales libres $\left({ }^{\circ} \mathrm{OH} \mathrm{y} \bullet^{\bullet} \mathrm{O}_{2}{ }^{-}\right)$, así como por los huecos en la banda de valencia del fotocatalizador (Chong et al., 2010). No obstante, debido a la amplia variedad de moléculas orgánicas, el desempeño y el mecanismo de la fotodegradación dependerá de varios factores tales como:

1. La especiación del contaminante. Debido a que la fotocatálisis heterogénea es un proceso llevado a cabo en la superficie del fotocatalizador, es prioritario que el contaminante orgánico sea adsorbido en la superficie del sólido. La adsorción del compuesto sobre el catalizador (un óxido metálico o una nanopartícula metálica) ocurrirá mediante interacciones electrostáticas entre los grupos funcionales de la molécula orgánica y las cargas presentes en la superficie del fotocatalizador. Por ello, las moléculas orgánicas no polares serán pobremente adsorbidas en la superficie del sólido, mientras que ésta se maximizará en caso de probar moléculas polares o semipolares con grupos funcionales ionizables, como carboxilo, hidroxilo o amino.

2. El pH del medio. Dado que este parámetro define tanto el estado de ionización de las moléculas orgánicas como la carga en la superficie del fotocatalizador, el mejor desempeño en la reacción fotocatalítica podrá ser alcanzado cuando el catalizador y la molécula orgánica presenten cargas opuestas, o bien, cuando por lo menos uno de ellos esté en su punto de carga cero (Van Doorslaer et al., 2012).

3. La presencia de oxígeno disuelto en el agua. Debido a que la degradación fotocatalítica de los contaminantes orgánicos es un proceso de oxidación, llevado a cabo tanto por el hueco foto-formado, como por los radicales libres producidos en la banda de valencia, es necesario evitar la recombinación del par hueco-electrón. Una vía para evitar la recombinación es neutralizar el electrón foto-formado en la banda de conducción mediante la adición de un agente electrófilo. El oxígeno disuelto en el agua, además de secuestrar al electrón de la banda de conducción, es capaz de generar otras especies oxidantes como el peróxido de hidrógeno y radicales libres, tales como ${ }^{\circ} \mathrm{O}_{2}{ }^{-} \mathrm{y}^{\bullet} \mathrm{OH}$. Se ha reportado un considerable incremento de la actividad fotocatalítica cuando la suspensión se satura con oxígeno disuelto, mientras que su ausencia lleva al detrimento en el rendimiento de la reacción (Van Doorslaer et al., 2012).

4. La composición química del agua. Diversos componentes disueltos en 
el agua tienen efecto en el proceso de fotocatálisis, algunos de ellos pueden acelerar la reacción, tales como la presencia del ion nitrato. Otros componentes pueden impactar negativamente en la fotocatálisis, como el ion carbonato, el cual secuestra a los radicales libres fotoformados, o a la materia orgánica, que impide la dispersión de la luz a través del agua (Wang et al., 2015).

5. La presencia concomitante de otros compuestos orgánicos. Cuando están presentes varios compuestos orgánicos al mismo tiempo en el sistema de reacción fotocatalítica, se pueden presentar fenómenos de competencia por los sitios de adsorción en la superficie del catalizador, o por las especies oxidantes formadas durante el proceso. La presencia de otras sustancias orgánicas en concentraciones más altas que el contaminante objetivo es un obstáculo importante en la reacción de fotocatálisis; esto ocurre especialmente cuando se trata agua con alto contenido de material orgánico, como el agua residual (Wang et al., 2015).

\section{Transformación de iones inorgánicos tóxicos en especies inocuas}

Entre los contaminantes de carácter inorgánico en el agua se pueden mencionar los compuestos nitrogenados, tales como el amonio y el nitrato, y los metales pesados. La conversión fotocatalítica del amonio $\left(\mathrm{NH}_{4}{ }^{+}\right)$en nitrógeno molecular $\left(\mathrm{N}_{2}\right)$ entraña el reto de establecer una ruta selectiva para evitar la producción de nitrato $\left(\mathrm{NO}_{3}{ }^{-}\right)$. La reducción del amonio es una reacción llevada a cabo en medio alcalino, a través de la transformación del amonio en amoniaco $\left(\mathrm{NH}_{3}\right)$ y su posterior reducción a nitrógeno molecular. Debido a que las nanopartículas de metales de Pt, Pd y Rh tienen energía de adsorción de nitrógeno alta afinidad por el amoniaco, éstas son comúnmente usadas para llevar a cabo el proceso de reducción fotocatalítica (De Vooys et al., 2001).

La transformación del amoniaco en nitrógeno molecular ocurre a través de una serie de reacciones de oxidación en presencia tanto de los huecos foto-formados en la banda de valencia del semiconductor como por radicales $\cdot \mathrm{OH}$, tal y como se muestra en las ecuaciones 6,7 y 8.

$$
\begin{aligned}
& \mathrm{NH}_{3(\mathrm{ads})}+\cdot \mathrm{OH} \longleftrightarrow \mathrm{NH}_{2(\mathrm{ads})}+\mathrm{H}_{2} \mathrm{O} \\
& \mathrm{NH}_{2(\mathrm{ads})}+\cdot \mathrm{OH} \longleftrightarrow \mathrm{NH}_{(\mathrm{ads})}+\mathrm{H}_{2} \mathrm{O} \\
& \mathrm{NH}_{(\mathrm{ads})}+\cdot \mathrm{OH} \longleftrightarrow \mathrm{N}_{(\mathrm{ads})}+\mathrm{H}_{2} \mathrm{O}
\end{aligned}
$$

Posteriormente, se produce el nitrógeno molecular sobre la superficie de las nanopartículas metálicas por la unión de los átomos de nitrógeno mediante reacciones con el hueco foto-formado (ecuaciones 9 y 10). 
FIGURA 3. Conversión fotocatalítica de $\mathrm{NO}_{3}{ }^{-}$en $\mathrm{N}_{2}$ empleando $\mathrm{TiO}_{2}$ modificado en superficie con nanopartículas bimetálicas de aleación de $\mathrm{Pd}-\mathrm{Cu}$.

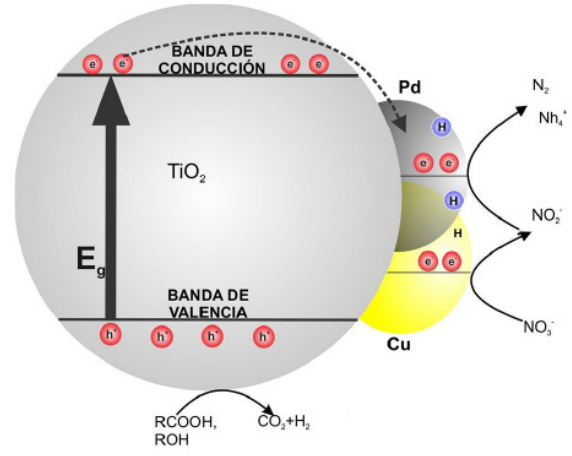

Fuente: Elaboración propia.

$$
\begin{gathered}
\mathrm{NH}_{\mathrm{x}(\mathrm{ads})}+\mathrm{NH}_{\mathrm{y}(\mathrm{ads})} \longleftrightarrow \mathrm{N}_{2} \mathrm{H}_{\mathrm{x}+\mathrm{y}(\mathrm{ads})} \\
\mathrm{NH}_{\mathrm{x}+\mathrm{y}(\mathrm{ads})}+(\mathrm{x}+\mathrm{y}) \mathrm{h}^{+} \longleftrightarrow \mathrm{N}_{2(\mathrm{ads})}+\mathrm{H}^{+}
\end{gathered}
$$

Esta ruta de degradación ha sido reportada por Altomare y Selli (2013), quienes sostienen que la transformación del ion amonio en $\mathrm{N}_{2}$ tiene alta selectividad al probar $\mathrm{TiO}_{2}$ modificado en superficie con nanopartículas de $\mathrm{Pd}$. Debido a la alta selectividad de la reacción cuando se emplea este fotocatalizador se observa una baja producción de intermediaros, tales como los iones nitrato y nitrito, lo cual le confiere un valor agregado desde el punto de vista ambiental.

En lo que respecta a la reducción fotocatalítica del ion $\mathrm{NO}_{3}{ }^{-}$hacia $\mathrm{N}_{2}$ los estudios hasta ahora reportados señalan que esta reacción de fotocatálisis puede llevarse a cabo empleando $\mathrm{TiO}_{2}$ modificado en superficie con nanopartículas de $\mathrm{Pd}$ o nanopartículas bi-metálicas de $\mathrm{Pt}-\mathrm{Cu}, \mathrm{Pd}-\mathrm{Cu}$ y $\mathrm{Ni}-\mathrm{Cu}$ (Gao et al., 2004). En particular, el uso de nanopartículas de $\mathrm{Pd}-\mathrm{Cu}$ sobre $\mathrm{TiO}_{2}$ ha mostrado incrementar en casi un $100 \%$ la selectividad de la reacción de conversión de $\mathrm{NO}_{3}{ }^{-}$y $\mathrm{NO}_{2}{ }^{-}$en $\mathrm{N}_{2}$, sin la producción de $\mathrm{NH}_{4}{ }^{+}$(Soares et al., 2014). En la figura 3 se muestran los mecanismos de reacción seguidos en la reducción fotocatalítica de $\mathrm{NO}_{3}{ }^{-}$al emplear semiconductores modificados en superficie con nanopartículas mono-metálicas y bi-metálicas.

La reducción fotocatalítica de las especies de nitrógeno precisa de la presencia simultánea de una molécula orgánica en la solución para consumir los huecos foto-formados y con ello evitar la reoxidación de los intermediarios; este componente orgánico se conoce como agente de sacrificio. Diferentes moléculas orgánicas han sido empleadas como agentes de sacrificio para la reducción fotocatalítica del $\mathrm{NO}_{3}{ }^{-}$, tales como el oxalato de sodio, el benceno (Li et al., 2010), el ácido fórmico, el ácido acético y otras moléculas orgánicas (Zhang et al., 2005). 
FIGURA 4. Inactivación y muerte de una célula bacteriana mediante fotocatálisis heterogénea empleando un semiconductor modificado en superficie con nanopartículas metálicas.

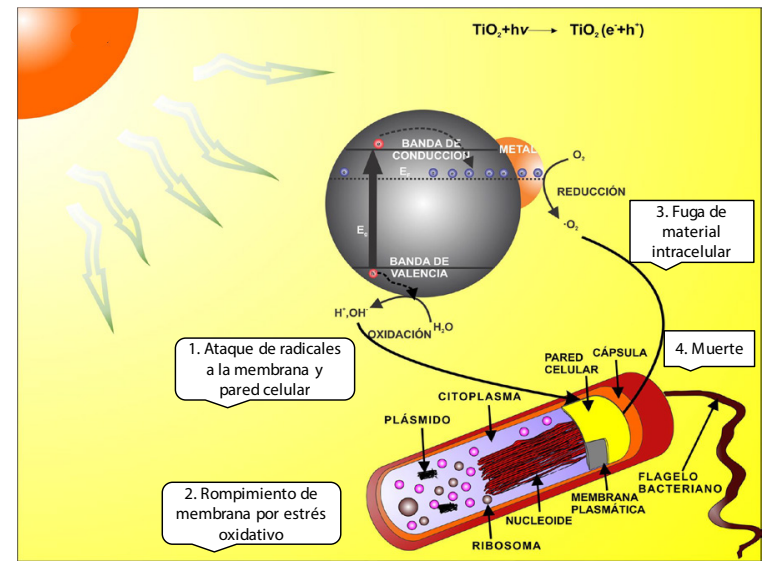

Fuente: Elaboración propia.

La remoción de metales pesados $\left(\mathrm{Pb}^{2+}, \mathrm{Cr}^{6^{+}}, \mathrm{As}^{3^{+}}\right.$, etc.) en agua puede lograrse por medios fotocatalíticos a través del fotodepósito reductivo de los metales pesados sobre la superficie de $\mathrm{TiO}_{2}$. Este proceso implica que durante la irradiación con luz UV, los metales pesados son depositados e inmovilizados en la superficie del semiconductor. Para este sistema de tratamiento, se reportan tasas de remoción de hasta $41 \%$ de $\mathrm{Pb}$, tras 17 horas de irradiación (Kabra et al., 2008). Otros metales pesados como el arsénico trivalente puede ser oxidado hacia su especie inocua $\mathrm{As}^{5^{+}}$mediante fotocatálisis heterogénea empleando $\mathrm{TiO}_{2}$ (Ryu y Choi, 2004).

\section{Desinfección del agua}

La fotocatálisis heterogénea ha sido también empleada para inactivar y aniquilar diferentes tipos de microrganismos patógenos en el agua, desde bacterias relativamente inocuas como E. coli, la cual es parte de la flora intestinal, hasta organismos de alta patogenicidad como el Vibrio chlorae, el cual es el causante del cólera (Das et al., 2015). Al igual que para la degradación de contaminantes orgánicos, la desinfección fotocatalítica del agua ocurre mediante reacciones de oxidación. Tres mecanismos han sido propuestos para la inactivación de los microrganismos durante el proceso fotocatalítico, estos son: a) la interrupción de la replicación celular por daño en el ADN bacteriano causado por radicales ${ }^{\circ} \mathrm{OH}$ (Hirakawa et al., 2004); b) daño estructural en la membrana y la pared celular por estrés oxidativo (Saito et al., 1992), lo cual lleva a la fuga de material intracelular (Wu et al., 2010), y, c) el decaimiento de la respiración celular por la oxidación intracelular de la acetil coenzima A (Matsunaga et al., 1985). En la figura 4, se muestran de manera 
esquemática estos mecanismos. Aun cuando existe poca evidencia acerca de cuál de estos procesos es el que lleva a la efectiva muerte celular, una buena parte de la literatura apunta a los daños en la membrana y la pared celular como la causa más plausible (Lu et al., 2003). Los fotocatalizadores más empleados en los procesos de desinfección son el $\mathrm{TiO}_{2}$ y las nanopartículas de plata. La plata, como metal noble, es un agente bactericida empleado para la desinfección de agua a nivel comercial, debido a su amplio espectro de acción contra bacterias, hongos y virus y a su baja toxicidad en mamíferos. Las nanopartículas de plata pueden ligarse a la membrana y a la pared celular y causar oquedades que desestabilizan el funcionamiento celular y llevan a la inactivación del patógeno (Rai et al., 2009). Adicionalmente, las nanopartículas de plata pueden dañar la estructura del ADN bacteriano impidiendo la replicación celular (Jung et al., 2008). Adicionalmente, otros metales como el oro y el platino también se han empleado como catalizadores en procesos de desinfección fotocatalítica, aunque con menores rendimientos que las nanopartículas de plata (Chen et al., 2010; Su et al., 2012).

\section{Modificación de los materiales nanoestructurados para la eliminación de contaminantes en agua}

Muchos semiconductores en forma nanométrica (tales como $\mathrm{TiO}_{2} \mathrm{ZnO}$, $\mathrm{ZrO}_{2}, \mathrm{CdS}, \mathrm{MoS}_{2}, \mathrm{Fe}_{2} \mathrm{O}_{3}, \mathrm{WO}_{3}$, etc.) han sido examinados y usados como fotocatalizadores para la degradación de contaminantes en agua (Auglugiaro et al., 2010; Durán-Álvarez et al., 2014; Hou y Cronin, 2013; Pan et al., 2012; Zhou et al., 2012). De estos semiconductores, $\mathrm{el} \mathrm{TiO}_{2}$ ha sido el más ampliamente utilizado. Sin embargo, este semiconductor presenta la desventaja de activarse principalmente con luz ultravioleta, lo cual lo hace inadecuado para el tratamiento de agua con luz solar, pues sólo $4 \%$ del total de la radiación solar troposférica está en el espectro UV. Lo anterior plantea la necesidad de modificar el fotocatalizador para ser activo en el espectro de luz visible, así como mejorar su desempeño fotocatalítico en la zona UV del espectro. Diferentes modificaciones estructurales de los nanomateriales fotocatalíticos se han propuesto para lograr tales mejoras, a continuación se mencionan algunas de ellas.

\section{Modificación superficial de semiconductores con nanopartículas metálicas}

El depósito de pequeñas cantidades de metales nobles en forma de nanopartículas sobre la superficie de un semiconductor puede incrementar de manera importante su fotoactividad, siempre y cuando estas nanoestructuras presenten propiedades plasmónicas (Cao et al., 2011; Zhou et al., 2012; Hou y Cronin, 2013). Cuando los semiconductores modificados con nanopartículas metálicas se irradian con luz de longitud de onda correspondiente a la banda 
FIGURA 5. Esquema de migración de electrones y producción de radicales libres en el proceso fotocatalítico usando semiconductores modificados en superficie con nanopartículas metálicas.

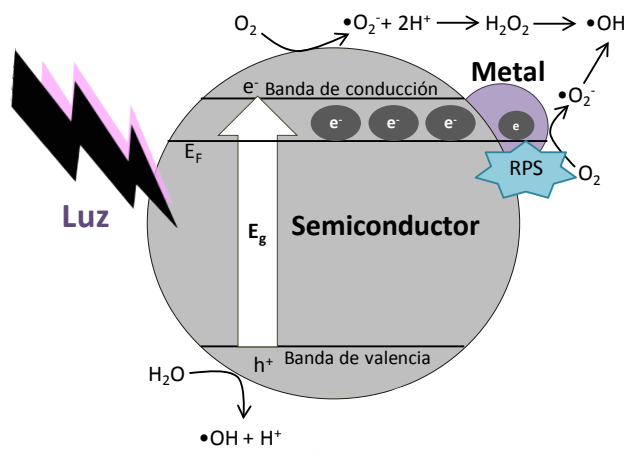

Fuente: Elaboración propia.

del plasmón, los electrones en la banda de conducción de la nanopartícula metálica son excitados y pueden migrar hacia la banda de conducción del semiconductor, o bien, reducir a las especies químicas adsorbidas en la superficie de la nanopartícula (Li et al., 2008). La presencia de nanopartículas metálicas en la superficie de un semiconductor ayuda también a evitar la recombinación del par hueco-electrón, debido a que las nanopartículas metálicas actúan como una trampa eficiente de electrones, la cual secuestra a los electrones presentes en la banda de valencia, promoviendo la eficiente separación de cargas, incrementando el tiempo de actividad del fotocatalizador y aumentando con ello la eficiencia de la reacción de fotocatálisis. En la figura 5 se muestra de manera esquemática la transferencia de electrones y la producción de radicales libres en el sistema semiconductor-nanopartícula metálica.

Las propiedades morfológicas tanto de la nanopartícula metálica como del semiconductor impactan en el desempeño fotocatalítico del material conjunto. Como se mencionó previamente, el tamaño de las nanopartículas del metal noble es de gran relevancia en el desempeño fotocatalítico del material modificado, ya que el incremento en el tamaño de las nanopartículas metálicas disminuye la expresión de la resonancia superficial del plasmón. La figura 6 muestra la eficiencia de mineralización del metil tert-butil éter, un aditivo empleado para incrementar el octanaje de la gasolina sin plomo, utilizando $\mathrm{TiO}_{2}$ modificado en superficie con nanopartículas de oro. Se observa que un incremento en el tamaño de las nanopartículas de oro resulta en el decaimiento en la tasa de degradación del compuesto orgánico (RodríguezGonzález et al., 2008). Al igual que el tamaño de las nanopartículas metálicas, otros parámetros como la forma o la dispersión de las nanopartículas metálicas en la superficie del semiconductor tienen un efecto importante en el desempeño del fotocatalizador para la degradación de contaminantes en agua. 
FIGURA 6. Eficiencia de la remoción de metil tert-butil éter, medido por determinación de carbono orgánico total (TOC) en función del tamaño de partícula de oro soportado en semiconductores basados en $\mathrm{TiO}_{2}$.

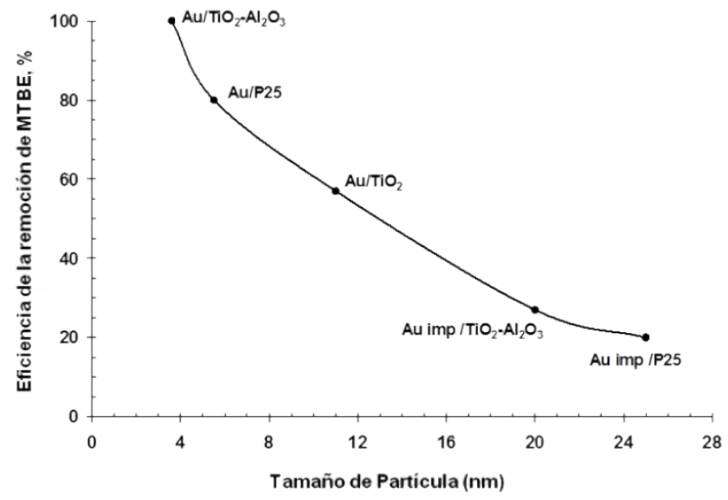

Fuente: Rodríguez-González et al., (2008).

En términos generales, la eficiencia de remoción de contaminantes en agua es significativamente mayor al emplear los semiconductores modificados en superficie con nanopartículas metálicas que los materiales sin modificar. Para la remoción de contaminantes orgánicos en agua, los semiconductores modificados en superficie con nanopartículas de oro han mostrado ser una de las mejores opciones, al reportar incrementos en la velocidad de degradación de diversas moléculas en un factor de 10 en comparación con el material sin modificar. Adicionalmente, es posible alcanzar la completa mineralización de moléculas recalcitrantes como los polifenoles en la mitad del tiempo que requieren los semiconductores sin modificar (DuránÁlvarez et al., 2014).

En el caso de los contaminantes inorgánicos, las reducciones selectivas de especies del nitrógeno, como el amonio, son rápidamente alcanzadas al emplear $\mathrm{TiO}_{2}$ modificado en superficie con nanopartículas de Pd o con nanopartículas bi-metálicas de $\mathrm{Au}-\mathrm{Pd}$ (Altomare y Selli, 2013). La baja producción de intermediaros, como los iones nitrato y nitrito, le dan a este catalizador un valor agregado desde el punto de vista ambiental. Estos niveles de conversión con altas tasas de selectividad no pueden ser alcanzados con semiconductores no modificados.

Como se mencionó previamente, la fotocatálisis heterogénea usando nanopartículas de plata resulta en altas tasas de desinfección del agua. Al depositar estas nanopartículas sobre semiconductores como el $\mathrm{TiO}_{2}$ se obtienen tasas de desinfección tan altas como de un 100\% tras sólo 2 horas de irradiación solar (Mirkhani et al., 2009). Semiconductores modificados en superficie con diferentes metales han sido empleados en el proceso de desinfección fotocatalítica de agua, aunque con resultados menos alentadores que lo reportado para $\mathrm{TiO}_{2}$ con depósito de plata. Por ejemplo, Chen et al. (2010) 
FIGURA 7. Esquema del proceso de fotocatálisis en un composito ternario $\mathrm{Au} / \mathrm{TiO}_{2} / \mathrm{WO}_{3}$.

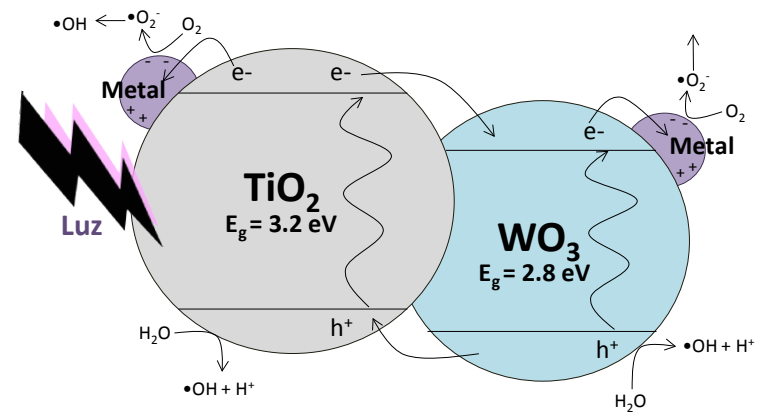

Fuente: Elaboración propia.

encontraron que al emplear $\mathrm{Au} / \mathrm{TiO}_{2}$ en la desinfección fotocatalítica de E. coli, un $50 \%$ de inhibición puede ser alcanzado tras 6 horas de irradiación con luz visible. Suri et al. (2012) probaron $\mathrm{TiO}_{2}$ nanoparticulado y modificado en superficie con Pt y Ag para la inactivación de la bacteria E. coli en agua potable, agua de río y agua residual tratada. Los autores reportan que tras 120 minutos de irradiación solar sólo se logró inactivar una tercera parte de la población microbiana en las muestras de agua. $\mathrm{El} \mathrm{TiO}_{2}$ modificado con plata resultó más eficiente en la inactivación de la bacteria que el semiconductor platinizado.

\section{Compositos binarios o ternarios}

Una forma de promover una mayor separación del par hueco-electrón es la síntesis de compositos binarios, es decir, combinando dos semiconductores, tales como CdS y $\mathrm{TiO}_{2}$ (Serpone et al., 1995) o $\mathrm{WO}_{3}$ y WS${ }_{2}$ (Di-Paola et al., 1999). Los compositos binarios son modificados por el depósito de nanopartículas metálicas para crear compositos ternarios. La combinación de dos semiconductores con diferentes energías de banda prohibida permite la transferencia vectorial de los electrones y los huecos foto-formados entre los óxidos metálicos, lo cual reduce la recombinación del par hueco-electrón y acelera las reacciones de degradación en la superficie del composito. El funcionamiento de esta clase de compositos múltiples, usando como ejemplo el material $\mathrm{Au} / \mathrm{TiO}_{2} / \mathrm{WO}_{3}$ se muestra de manera esquemática en la figura 7. Esta clase de compositos ternarios ha mostrado ser más eficiente en la mineralización de contaminantes orgánicos recalcitrantes, como el plastificante bisfenol A, empleando luz solar que los componentes individuales (Kovacs et al., 2014). En el caso de la eliminación de bacterias, el composito ternario $\mathrm{Ag}-\mathrm{AgBr}-\mathrm{Bi}_{2} \mathrm{WO}_{6}$ ha mostrado muy altas tasas de inactivación de la bacteria E. coli K-12. La completa inactivación de la bacteria (a una concentración inicial de $5 \times 10^{7}$ unidades formadoras de colonias $/ \mathrm{mL}$ ) se alcanzó tras apenas 
15 minutos de irradiación con luz visible. La completa destrucción de las células bacterias se atribuyó a la elevada producción de radicales ${ }^{\bullet} \mathrm{OH}$ y huecos foto-formados en la superficie del composito (Zhang et al., 2009).

\section{Comentarios finales}

La fotocatálisis heterogénea es un proceso de oxidación avanzada que ha sido implementado de manera exitosa en la mayoría de los casos para la eliminación de contaminantes en el agua. Este proceso puede degradar contaminantes orgánicos hasta sus componentes minerales en periodos cortos de tiempo; también puede llevar a cabo reacciones selectivas de oxidación o reducción de contaminantes inorgánicos con altos niveles de selectividad, así como inactivar y eliminar microrganismos patógenos en el agua, desinfectándola eficientemente. Las reacciones de oxidación y reducción en las que se basa el proceso fotocatalítico pueden ser potenciadas al modificar la estructura y morfología de los nanomateriales empleados como fotocatalizadores. El empleo de nanopartículas con alta área superficial, así como la modificación en superficie de semiconductores con nanopartículas metálicas han mostrado excelentes resultados para la activación del fotocatalizador en el espectro de luz solar. Debido al fenómeno de resonancia de plasmón de superficie es posible llevar a cabo reacciones fotocatalíticas de descontaminación de agua con luz solar y empleando semiconductores de baja toxicidad y amplio valor de banda prohibida como el $\mathrm{TiO}_{2}$. El depósito de nanopartículas metálicas sobre semiconductores incrementa efectivamente la rapidez de las reacciones fotocatalíticas de interés ambiental, como la inactivación de microrganismos patógenos, la transformación de agentes inorgánicos tóxicos en especies inocuas y la degradación de contaminantes orgánicos.

Al emplear diferentes arreglos de semiconductores modificados en superficie con nanopartículas metálicas es posible dar un mayor aprovechamiento a las reacciones de oxidación-reducción que ocurren en la superficie del catalizador. Ello puede llevarse a cabo a través del acoplamiento de reacciones como: a) la reducción de especies iónicas como nitratos y la oxidación de moléculas orgánicas, y, b) la inactivación de microrganismos patógenos y la remoción de algunos subproductos de desinfección - como los trihalometanos. Los estudios futuros deberán enfocarse en el desarrollo de sistemas fotocatalíticos para la depuración efectiva de aguas residuales tratadas o agua para consumo humano en volúmenes ambientalmente relevantes. Para ello será necesario desarrollar reactores en donde se traten en un solo paso, o de manera continua, grandes volúmenes de agua con luz solar, empleando tiempos de reacción cortos. 


\section{Referencias}

Akpan, U. G., y B. H. Hameed (2009). Parameters affecting the photocatalytic degradation of dyes using TiO-based photocatalysts: A review. Journal of Hazardous Materials, vol. 170, núm. 2: 520-529.

Altomare, M., Selli, E. (2013). Effects of metal nanoparticles deposition on the photocatalytic oxidation of ammonia in $\mathrm{TiO}_{2}$ aqueous suspensions. Catalysis Today, vol. 209: 127.

Astruc, D. (2008). Nanoparticles and catalysis. NJ, USA: Wiley.

Augugliaro, V., Loddo, V., Pagliaro, M., Palmisano, G., Palmisano, L. (2010). Clean by light irradiation. Practical applications of supported $\mathrm{TiO}_{2}$. Cambridge, UK: RSC.

Cao, S. W., Yin, Z., Barber, J., Boey, F. Y. C., Loo, S. C. J., Xue, C. (2011). Preparation of $\mathrm{Au}-\mathrm{BiVO}_{4}$ heterogeneous nanostructures as highly efficient visible-light photocatalysts. ACS Applied Materials and Interfaces, vol. 4, núm. 1: 418.

Chen, J., Albella, P., Pirzadeh, Z., Alonso-González, P., Huth, F., Bonetti, S., Bonanni, V., Akerman, J., Nogués, J., Vavassori, P., Dmitriev, A., Aizpurua, J., Hillenbrand, R. (2011). Plasmonic nickel nanoantennas. Small, vol. 7, núm. 16: 2341.

Chen, S. F., Li, J. P., Qian, K., Xu, W. P., Lu, Y., Huang, W. X., Yu, S. H. (2010). Large scale photochemical synthesis of $\mathrm{M} @ \mathrm{TiO}_{2}$ nanocomposites $(\mathrm{M}=\mathrm{Ag}, \mathrm{Pd}, \mathrm{Au}$, $\mathrm{Pt}$ ) and their optical properties, $\mathrm{CO}$ oxidation performance, and antibacterial effect. Nano Research, vol. 3, núm. 4: 244.

Chong, M. N., Bo J., Chow C. W. K., Saint C. (2010). Recent developments in photocatalytic water treatment technology: a review. Water Research, vol. 44, núm. 10: 2997.

Comninellis, C., Kapalka, A., Malato, S., Parsons, S. A., Poulios, I., Mantzavinos D. (2008). Advanced oxidation processes for water treatment: Advances and trends for R\&D. Journal of Chemical Technology and Biotechnology, vol. 83, núm. 6: 769.

Cushing, S. K., Li, J., Meng, F., Sentry, T. R., Suri, S., Zhi, M. (2012). Photocatalytic activity enhanced by plasmonic resonant energy transfer from metal to semiconductor. The Journal of the American Chemical Society, vol. 134, núm. 36, 2: 15033.

Das, S., Sinha, S., Suar, M., Yun, S. I., Mishra, A., Tripathy, S. K. (2015). Solar-photocatalytic disinfection of Vibrio cholerae by using Ag@ZnO core-shell structure nanocomposites. Journal of Photochemistry and Photobiology B: Biology, vol. 142: 68.

Daughton, C. G. (2004). Non-regulated water contaminants: Emerging research. Environmental Impact Assessment Research, vol. 24, núm. 7: 711.

De Vooys, A. C. A., Koper, M. T. M., van Santen, R. A., van Veen, J. A. R. (2001). The role of adsorbates in the electrochemical oxidation of ammonia on noble and transition metal electrodes. Journal of Electroanalytical Chemistry, vol. 506, núm. 2: 127.

Deblonde, T., Cossu-Leguille, C., Hartemann, P. (2011). Emerging pollutants in wastewater: A review of the literature. International Journal of Hygiene and Environmental Health, vol. 214, núm. 6: 442. 
Di-Paola, A., Palmisano, L., Venezia, A. M., Augugliaro, V. (1999). Preparation and characterization of polycrystalline mixed $\mathrm{WO}_{3} / \mathrm{WS}_{2}$ powders. The Journal of Physical Chemistry B., vol. 103, núm. 39: 8236.

Durán-Álvarez, J. C., Zanella, R., Oros-Ruiz, S. (2014). Heterofgeneous gold catalysts and catalysis, cap. 7. Londres: The Royal Society of Chemistry.

Eustis, S., El-Sayed, M. A. (2006). Why gold nanoparticles are more precious than pretty gold: Noble metal surface plasmon resonance and its enhancement of the radiative and nonradiative properties of nanocrystals of different shapes. Chemical Society Reviews, vol. 35: 209.

Farré, M., Pérez, S., Kantiani, L., Barceló, D. (2008). Fate and toxicity emerging pollutants, their metabolites and transformation products in the aquatic environment. Trends in Analytical Chemistry, vol. 27, núm. 11: 991.

Gao, W., Jin, R., Chen, J., Guan, X., Zeng, H., Zhang, F., Guan, N. (2004). Titaniasupported bimetallic catalysts for photocatalytic reduction of nitrate. Catalysis Today, vol. 3, núm. 90: 331.

Gwak, J. H., Kim, S. J., Lee, M. (1998). Sol-gel preparation of $\mathrm{AuCu}$ and $\mathrm{Au}_{4} \mathrm{Cu}$ nanocluster alloys in silica thin films. The Journal of Physical Chemistry B., vol. 102, núm. 40: 7699.

Hashmi, A. S. K., Hutchings, G. J. (2006). Gold catalysis. Angewandte Chemie International Edition, vol. 45, núm. 47: 7896.

Hirakawa, K., Mori, M., Yoshida, M., Oikawa, S., Kawanishi, S. (2004). Photo-irradiated titanium dioxide catalyzes site specific DNA damage via generation of hydrogen peroxide. Free Radical Research, vol. 38, núm. 5: 439.

Hou, W., Cronin, S. B. (2013). A review of surface plasmon resonance-enhanced photocatalysis. Advanced Functional Materials, vol. 23, núm. 13: 1612.

Jung, W. K., Koo, H. C., Kim, K. W., Shin, S., Kim, S. H., Park, Y. H. (2008). Antibacterial activity and mechanism of action of the silver ion in Staphylococcus aureus and Escherichia coli". Applied and Environmental Microbiology, vol. 74, núm. 7: 2171.

Kabra, K., Chaudhary, R., Sawhney, R. L. (2008). Solar photocatalytic removal of Cu (II), Ni (II), Zn (II) and Pb (II): Speciation modeling of metal-citric acid complexes. Journal of Hazardous Materials, vol. 155, núm. 3: 424.

Jungwon, K. Choi, W. (2010). Hydrogen producing water treatment through solar photocatalysis. Energy \& Environmental Science, vol. 3, núm. 8: 1042.

Kovács, G., Baia, L., Vulpoi, A., Radu, T., Karácsonyi, E., Dombi, A., Hernádi, K., Danciu, V., Simon, S., Pap, Z. (2014). $\mathrm{TiO}_{2} / \mathrm{WO}_{3} / \mathrm{Au}$ nanoarchitectures' photocatalytic activity, from degradation intermediates to catalysts' structural peculiarities", Part I: Aeroxide P25 based composites. Applied Catalysis B: Environmental, vol. 147: 508.

Lapworth, D. J., Baran, N., Stuart, M. E., Ward, R. S. (2012). Emerging organic contaminants in groundwater: A review of sources, fate and occurrence. Environmental Pollution, vol. 163: 287.

Lastre, Acosta, A. M., Cruz-González, G., Nuevas-Paz, L., Jáuregui-Haza, N. J., Teixeira, A. C. S. C. (2014). Ultrasonic degradation of sulfadiazine in aqueous 
solutions. Environmental Science Pollution Research, vol. 22, núm 2 : 918.

Le, F., Brandl, D. W., Urzhumov, Y. A., Wang, H., Kundu, J., Halas, N. J. (2008). Metallic nanoparticle arrays: A common substrate for both surface-enhanced Raman scattering and surface-enhanced infrared absorption. ACS Nano, vol. 2, núm. 4: 707.

Li, L., Xu, Z., Liu, F., Shao, Y., Wang, J., Wan, H., Zheng, S. (2010). Photocatalytic nitrate reduction over $\mathrm{Pt}-\mathrm{Cu} / \mathrm{TiO}_{2}$ catalysts with benzene as hole scavenger. Journal of Photochemistry and Photobiology A: Chemistry, vol. 212, núm. 2: 113.

Li, M., Zhou, S., Zhang, Y., Chen, G., Hong, Z. (2008). One-step solvothermal preparation of $\mathrm{TiO}_{2} / \mathrm{C}$ composites and their visible-light photocatalytic activities. Applied Surface Science, vol. 254, núm. 13: 3762.

Lin, W. C., Yang, W. D., Huang, I. L., Wu, T. S., Chung, Z. J. (2009). Hydrogen production from methanol/water photocatalytic decomposition using $\mathrm{Pt} / \mathrm{TiO}_{2}-{ }_{\mathrm{x}} \mathrm{N}_{\mathrm{x}}$. Catalyst. Energy \& Fuels, vol. 23, núm. 4: 2192.

Lu, Z. X., Zhou, L., Zhang, Z. L., Shi, W. L., Xie, Z. X., Xie, H. Y., Pang, D. W., Shen, P. (2003). Cell damage induced by photocatalysis of $\mathrm{TiO}_{2}$ thin films. Langmuir, vol. 19, núm. 21: 876.

Malato, S., Fernández-Ibañez, P., Maldonado, M. I., Blanco, J., Gernjak, W. (2009). Decontamination and disinfection of water by solar photocatalysis: Recent overview and trends. Catalysis Today, vol. 147, núm. 1: 108.

Matsunaga, T., Tomoda, R., Nakajima, T., Wake, H. (1985). Photoelectrochemical sterilization of microbial cells by semiconductor powders. FEMS Microbiology Letters, vol. 29, núm. 1-2: 211.

Mirkhani, V., Tangestaninejad, S., Moghadam, M., Habibi, M. H., Vartooni, A. R. (2009). Photodegradation of aromatic amines by $\mathrm{Ag}-\mathrm{TiO}_{2}$ photocatalyst. Journal of the Iranian Chemical Society, vol. 6, núm. 4: 800.

Muneer, M., Bahnemann, D., Qamar, M., Tariq, M. A., Faisal M. (2005). Photocatalysed reaction of few selected organic systems in presence of titanium dioxide. Applied Catalysis A: General, vol. 289, núm. 2: 224.

Nagahama, Y., Nakamura, M., Kitano, T., Tokumoto, T. (2003). Sexual plasticity in fish: possible target of endocrine disruption action. Environmental Sciences: An International Journal of Environmental Physiology and Toxicology, vol. 11, núm. 1: 73.

Oller, I., Malato, S., Sánchez-Pérez J. A. (2011). Combination of advanced oxidation processes and biological treatments for wastewater decontamination-a review. Science of the Total Environment, vol. 409, núm. 20: 4141.

Osterloh, F. E. (2008). Inorganic materials as catalysts for photochemical splitting of water. Chemistry of Materials, vol. 20, núm. 1: 35-54.

Pal, A., Gin, K. Y. H., Lin, A. Y. C., Reinhard, M. (2010). Impacts of emerging organic contaminants on freshwater resources: A review of recent occurrences, sources, fate and effects. Science of the Total Environment, vol. 408, núm. 24: 6062.

Pan, Y., Deng, S., Polavarapu, L., Gao, N., Yuan, P., Sow, C. H. (2012). Plasmon- 
enhanced photocatalytic properties of $\mathrm{Cu}_{2} \mathrm{O}$ nanowire au nanoparticle assemblies. Langmuir, vol. 28, núm. 33: 12304.

Patsoura, A., Kondarides, D. I., Verykios X. E. (2007). Photocatalytic degradation of organic pollutants with simultaneous production of hydrogen. Catalysis Today, vol. 124, núm. 3: 94.

Polshettiwar, V., Asefa, T. (2013). Nanocatalysis: Synthesis and Applications. NJ, USA: Wiley.

Pradeep, T. (2009). Noble metal nanoparticles for water purification: a critical review. Thin Solid Films, vol. 517, núm. 24: 6441.

Primo, A., Corma, A., García, H. (2011). Titania supported gold nanoparticles as photocatalyst. Physical Chemistry Chemical Physics, vol. 13, núm. 3: 886.

Rai, M., Yadav, A., Gade, A. (2009). Silver nanoparticles as a new generation of antimicrobials. Biotechnology Advances, vol. 27, núm. 1: 76.

Rauf, M. A., Ashraf S. S. (2009). Fundamental principles and application of heterogeneous photocatalytic degradation of dyes in solution. Chemical Engineering Journal, vol. 151, núm. 1: 10.

Rodríguez-González, V., Zanella, R., del-Ángel, G., Gómez, R. (2008). MTBE visiblelight photocatalytic decomposition over $\mathrm{Au} / \mathrm{TiO} \mathrm{O}_{2}$ and $\mathrm{Au} / \mathrm{TiO}_{2}-\mathrm{Al}_{2} \mathrm{O}_{3}$ sol-gel prepared catalysts. Journal of Molecular Catalysis A, vol. 281, núm. 1: 93.

Rodríguez-Mozaz, S., Chamorro, S., Marti, E., Huerta, B., Gros, M., Sánchez-Melsió, A., Borrego, C. M., Barceló, D., Balcázar, J. L. (2015). Occurrence of antibiotics and antibiotic resistance genes in hospital and urban wastewaters and their impact on the receiving river. Water Research, vol. 69: 234.

Ryu, J., Choi, W. (2004). Effects of $\mathrm{TiO}_{2}$ surface modifications on photocatalytic oxidation of arsenite: The role of superoxide radicals. Environmental Science and Technology, vol. 38, núm. 10: 2928.

Saito, T., Iwase, T., Horie, J, Morioka, T. (1992). Mode of photocatalytic bactericidal action of powdered semiconductor $\mathrm{TiO}_{2}$ on mutans Streptococci. Journal of Photochemistry and Photobiology B: Biology, vol. 14, núm. 4: 369.

Serpone, N., Maruthamuthu, P., Pichat, P., Pelizzetti, E., Hidaka, H. (1995). Exploiting the interparticle electron transfer process in the photocatalysed oxidation of phenol, 2-chlorophenol and pentachlorophenol: Chemical evidence for electron and hole transfer between coupled semiconductors. Journal of Photochemistry and Photobiology A: Chemistry, vol. 85, núm. 3: 247.

Soares, O. S. G. P., Pereira, M. F. R., Örfao, J. M. M., Faria, J. L., Silva, C. G. (2014). Photocatalytic nitrate reduction over $\mathrm{Pd}-\mathrm{Cu} / \mathrm{TiO}_{2}$. Chemical Engineering Journal, vol. 251: 123.

Su, R., Tiruvalam, R., He, Q., Dimitratos, N., Kesavan, L., Hammond, C., López-Sánchez, J. A., Bechstein, R., Kiely, C. J., Hutchings, G. J., Besenbacher, F. (2012). Promotion of phenol photodecomposition over $\mathrm{TiO}_{2}$ using $\mathrm{Au}, \mathrm{Pd}$ and $\mathrm{Au}-\mathrm{Pd}$ nanoparticles. ACS Nano, vol. 6, núm. 7: 6284.

Suri, R. P., Thornton, H. M., Muruganandham, M. (2012). Disinfection of water using Pt-and Ag-doped $\mathrm{TiO}_{2}$ photocatalysts. Environmental Technology, vol. 33, núm. 14: 1651. 
Van Doorslaer, X., Heynderickx, P. M., Demeestere, K., Debevere, K., Van Langenhove, H., Dewulf, J. (2012). $\mathrm{TiO}_{2}$ mediated heterogeneous photocatalytic degradation of moxifloxacin: operational variables and scavenger study. Applied Catalysis B: Environmental, vol. 111: 150.

Wang, D., Li, Y., LiPuma, G., Wang, C., Wang, P., Zhang, W., Wan, Q. (2015). Mechanism and experimental study on the photocatalytic performance of $\mathrm{Ag} / \mathrm{AgCl} @$ chiral $\mathrm{TiO}_{2}$ nanofibers photocatalyst: The impact of wastewater components. Journal of Hazardous Materials, vol. 285: 277.

Wu, P. G., Imlay, J. A., Shang, J. K. (2010). Mechanism of Escherichia coli inactivation on palladium-modified nitrogen-doped titanium dioxide. Biomaterials, vol. 31, núm. 29: 7526.

You, J. B., Zhang, X. W., Dong, J. J., Song, X. M., Yin, Z. G., Chen, N. F., Yan, H. (2009). Localized-surface-plasmon enhanced the $357 \mathrm{~nm}$ forward emission from $\mathrm{ZnMgO}$ films capped by Pt nanoparticles. Nanoscale Research Letters, vol. 4, núm. 10: 1121.

Zhang, F., Jin, R., Chen, J., Shao, C., Gao, W., Li, L., Guan, N. (2005). High photocatalytic activity and selectivity for nitrogen in nitrate reduction on $\mathrm{Ag} / \mathrm{TiO}_{2}$ catalyst with fine silver clusters. Journal of Catalysis, vol. 232, núm. 2: 424.

Zhang, H., Chen, G. (2009). Potent antibacterial activities of $\mathrm{Ag} / \mathrm{TiO}_{2}$ nanocomposite powders synthesized by a one-pot sol-gel method. Environmental Science and Technology, vol. 43, núm. 8: 2905.

Zhou, X., Liu, G., Yu, J., Fan, W. (2012). Surface plasmon resonance-mediated photocatalysis by noble metal-based composites under visible light. Journal of Materials Chemistry, vol. 22, núm. 40: 21337.

Zhu, H., Chen, X., Zheng, Z., Ke, X., Jaatinen, E., Zhao, J., Guo, C., Xie, T., Wang, D. (2009). Mechanism of supported gold nanoparticles as photocatalysts under ultraviolet and visible light irradiation. Chemical Communications, vol. 48: 7524.

Zou, S. I., Schatz, G. C. (2005). Silver nanoparticle array structures that produce giant enhancements in electromagnetic fields. Chemical Physics Letters, vol. 403, núm. 1-3: 62. 